\title{
COMPACTIFICATIONS WHOSE REMAINDERS ARE RETRACTS
}

\author{
GARY D. FAULKNER
}

(Communicated by Doug W. Curtis)

\begin{abstract}
This paper is concerned with compactifications in which the remainder is a (neighborhood) retract. Two theorems which characterize such compactifications are proved here. One of the characterizations is in terms of singular functions and the other in terms of projections on spaces of continuous real valued functions.
\end{abstract}

The notion of the singular set of a mapping was defined and investigated by G. T. Whyburn [16] and George L. Cain, Jr. [1, 2]. Later it was realized that singular sets could be used to study compactifications $[\mathbf{3}, \mathbf{4}, \mathbf{5}]$. Many of the compactifications of a space arise naturally as "singular compactifications" and many of the theorems characterizing remainders in compactifications use singular mappings $[\mathbf{4}, \mathbf{5}]$. In this paper we generalize the notion of singular compactifications to remove a defect that unnecessarily restricts their applicability. We then characterize compactifications of this type in terms of the existence of retractions and neighborhood retractions from the compactification onto its remainder.

The retraction associated with a compactification of this type can be used to construct a projection from the Banach space of continuous functions which extend to the compactification onto the subspace consisting of functions which vanish at infinity. The result of $R$. S. Phillips [14] that $c_{0}$ is not complemented in $l_{\infty}$ is actually a theorem about spaces of continuous functions determined by compactifications in this manner. We will characterize compactifications in which the remainder is a retract in terms of the existence of such projections. Note $l_{\infty} \cong C(\beta \mathbf{N})$ and $c_{0} \cong C_{\infty}(\mathbf{N})$ where $\beta \mathbf{N}$ is the Stone-Cech compactification of $\mathbf{N}$ and $C_{\infty}(\mathbf{N})$ is the functions which vanish at infinity on $\mathbf{N}$. The one-point compactification of a space $X, \omega X$, is a singular compactification, and $C_{\infty}(X)$ is a subspace of $C(\omega X)$ of codimension one. Thus, as a result of Phillip's theorem, there can be no projection from $l_{\infty}$ onto $C(\omega \mathbf{N})$. As we will see, this theorem holds not only for the one-point compactification, but for any singular compactification.

In all that follows all spaces will be locally compact and Hausdorff. For a given compactification $\alpha X$ of $X, C_{\alpha}(X)$ will denote the collection of all real valued continuous functions on $X$ which extend to $\alpha X$. For a space $X, C_{\infty}(X)$ will denote the real valued continuous functions on $X$ which vanish at infinity. In other words, $C_{\infty}(X)=\{f \in C(X): \forall \varepsilon>0, \exists$ a compact $K \subset X$ such that $|f(x)|<\varepsilon$ if $x \notin K\}$. If $X$ is a Banach space, then $X^{*}$ will denote its topological dual. If $A$ is a bounded linear operator on $X$, then $A^{*}$ will denote the adjoint of $A$. A projection $P: X \rightarrow X$

Received by the editors March 9, 1987 and, in revised form, May 26, 1987.

1980 Mathematics Subject Classification (1985 Revision). Primary 54D35, 54D40, 54C10, 47B38.

Key words and phrases. Compactification, remainder, singular mappings, projections. 
is a continuous linear mapping which satisfies $P^{2}=P$. The complementary projection associated with $P$ is $I-P$. A subspace $Y \subseteq X$ is complemented if and only if $Y$ is the range of a projection.

DEFINITION 1. Let $X$ be noncompact. Let $S$ be compact and let $f: X \rightarrow S=$ $\overline{f(X)}$ be continuous. Then $f$ is said to be singular if for each open $U \subseteq S, f^{-1}(U)$ is not contained in any compact subset of $X$. Note that this definition is different from that of [3], where a function was singular if its singular set is nonempty. In this paper we require that the singular set of the function be its entire range. This is consistent with later usage $[\mathbf{4}, \mathbf{1 1}]$.

DEFINITION 2. Let $X$ and $S$ be as in the previous definition and let $K \subseteq X$ be compact. A continuous function $f: X \backslash K \rightarrow S=\overline{f(X \backslash K)}$ is weakly singular provided for all open $U \subseteq S, f^{-1}(U)$ is not contained in any compact subset of $X$.

Note that $f^{-1}(U)$ fails to have compact closure in $X$ rather than in $X \backslash K$. This is not the same as $f$ being a singular mapping from $X \backslash K$ to $S$ in the sense of Definition 1. Of course if $K=\Phi$ the two definitions coincide.

Let $f: X \backslash K \rightarrow S$ be weakly singular. Mimicking the construction in [4], we define a compact topology on $X \cup S$. A basis for the topology is as follows: For a point $p \in X$, an open neighborhood will be an ordinary open neighborhood in $X$. For a point $p \in S$, an open neighborhood will be of the form $U \cup f^{-1}(U) \backslash F$ where $F$ is any compact subset of $X$ and $U$ is any neighborhood of $p$ in $S$. This is clearly a basis for a compact topology on $X \cup S$. Since $X$ is locally compact, the topology is Hausdorff. In addition, the condition of singularity assures that $f^{-1}(U) \backslash F$ is not empty. From this it follows that $X$ is dense in $X \cup S$. We denote this compactification by $w_{f}(X, S)$ and call it the weakly singular compactification of $X$ generated by $f$. We continue the notation begun in [4] and denote by $X \cup_{f} S$ the compactification obtained when $f$ is singular. To distinguish these two cases we have introduced the somewhat clumsy "weakly singular".

THEOREM 1. Let $\alpha X$ be a compactification of $X$ then $\alpha X \backslash X$ is a (neighborhood) retract of $\alpha X$, if and only if $\alpha X$ is (weakly) singular.

PROOF. We will prove this for weakly singular compactifications. The case for singular compactifications appears in [11] and partially in [16] in a different context. Suppose $\alpha X$ is a weakly singular compactification. Thus $\alpha X=w_{f}(X, \alpha X \backslash X)$ for some weakly singular function $f$. Let the domain of $f$ be $U=X \backslash K$ where $K$ is compact in $X$ and let $W=\alpha X \backslash K$. Note that $W=U \cup(\alpha X \backslash X)$ is a neighborhood of $\alpha X \backslash X$ in $\alpha X$. Define $r: W \rightarrow \alpha X \backslash X$ by

$$
r(x)= \begin{cases}x & \text { if } x \in \alpha X \backslash X \\ f(x) & \text { if } x \in U\end{cases}
$$

We need only show that $r$ is continuous. If $V$ is an open set in $\alpha X \backslash X$, then $r^{-1}(V)=V \cup f^{-1}(V)$ is obviously open in $W$. Thus $\alpha X \backslash X$ is a neighborhood retract of $\alpha X$.

For the converse let us suppose that $\alpha X \backslash X$ is a neighborhood retract of $\alpha X$. In particular suppose that $U$ is a neighborhood of $\alpha X \backslash X$ and that $r: U \rightarrow \alpha X \backslash X$ is a retract. Note that $K=X \backslash U$ is compact. Let $f: U \backslash(\alpha X \backslash X) \rightarrow \alpha X \backslash X$ be given by $f=\left.r\right|_{U \backslash(\alpha X \backslash X)}=\left.r\right|_{X \backslash K}$. We must first show that $f$ is weakly singular. To this end let $V$ be a neighborhood in $\alpha X \backslash X$ and let $p \in V$. For each neighborhood 
$W$ of $p, r^{-1}(W)$ contains points of $X$. This is true by virtue of the fact that $X$ is dense in $\alpha X$, and hence $r(X)$ is dense in $\alpha X \backslash X$. Choose for each such $W$ a point $x_{W} \in r^{-1}(W) \cap X$. This is a net in $X$ which converges to $p$. If $f^{-1}(V)$ were contained in a compact subset of $X$, the net would cluster in $X$ which is impossible. Consequently $f$ is weakly singular.

It remains to show that $\alpha X$ is equivalent to $w_{f}(X, \alpha X \backslash X)$. For this it suffices to show that the identity mapping is continuous. This will be true if for each $U$ in $\alpha X \backslash X$ and compact $F$ in $X$, the set $U \cup f^{-1}(U) \backslash F$ is open in $\alpha X$. This set is nothing more than $r^{-1}(U) \backslash F$, which is clearly open.

COROLlARY 1. Let $\alpha X=w_{f}(X, S)$ be weakly singular (singular) and suppose that $\gamma X \leq \alpha X$, then $\gamma X$ is weakly singular (singular).

PROOF. Let $r$ be a neighborhood retraction (retraction) from $\alpha X$ to $\alpha X \backslash X$ and let $f$ denote the restriction of $r$ to that part of its domain which lies in $X$. Let $\pi$ be the quotient projection from $\alpha X$ to $\gamma X$. Define $r_{1}$ as follows:

$$
r_{1}(x)= \begin{cases}x & \text { if } x \in \gamma X \backslash X, \\ \pi \circ f(x) & \text { if } x \in \text { the domain of } f .\end{cases}
$$

We need only show that $r_{1}$ is continuous. Let $V$ be open in $\gamma X \backslash X$, then $r_{1}^{-1}(V)=$ $V \cup f^{-1} \circ \pi^{-1}(V)$. Since $\pi$ is a quotient map, $r_{1}^{-1}(V)$ will be open if and only if $\pi^{-1}\left(r_{1}^{-1}(V)\right)$ is open. But this set is $\pi^{-1}(V) \cup f^{-1}\left(\pi^{-1}(V)\right)$ which is a basic open set in $\alpha X$

COROLLARY 2. The collection of weakly singular (singular) compactifications forms a complete lower lattice.

PROOF. Any collection of compactifications has an infimum in the collection of all compactifications. By Corollary 1 this infimum is weakly singular (singular).

EXAMPLE 1. Let $S$ be a separable compact Hausdorff space and let $\left\{a_{1}, a_{2}, \ldots\right\}$ be a countable dense set. If $F$ is any compact subset of $\mathbf{N}$, then $F$ is finite. Define a function $f: \mathbf{N} \backslash F \rightarrow S$ so that each of $a_{k}$ is the image of infinitely many members of $\mathbf{N} \backslash F$. This function is clearly weakly singular. The compactification $w_{f}(\mathbf{N}, S)$ has $S$ as a remainder. Thus any separable compact Hausdorff space is the remainder of weakly singular compactification of $\mathbf{N}$. Clearly the remainder in any weakly singular compactification is a separable compact Hausdorff space so that this in fact characterizes the weakly singular remainders of $\mathbf{N}$. Since $\beta \mathbf{N} \backslash \mathbf{N}$ is not separable, $\beta \mathrm{N}$ is not weakly singular.

EXAMPLE 2 . The closed ball in $\mathbf{R}^{n}$ is a weakly singular compactification of the open ball $\left(\cong \mathbf{R}^{n}\right)$. Since the surface of the ball is not a retract of the closed ball this cannot be a singular compactification.

EXAMPLE 3. The two point compactification of $\mathbf{R}$ is weakly singular and not singular since the remainder is not connected. If $X$ consists of two copies of the long line with the initial points identified, then the two point compactification of $X$ is $\beta X$, which is weakly singular but not singular. In fact every compactification of a locally compact space $X$ having a finite remainder is weakly singular. To see this suppose that $\alpha X$ is a compactification of $X$ such that $\alpha X \backslash X$ consists of $n$-points $a_{1}, a_{2}, \ldots, a_{n}$. By Magill's [13] characterization of spaces with finite remainder, $X$ can be written as the union of a compact set $F$ and $n$ disjoint open sets $\left\{U_{k}\right\}_{k=1}^{n}$, 
where $U_{k} \cup F$ is not compact for any $1 \leq k \leq n$. Define $f: X \backslash F \rightarrow\left\{a_{1}, a_{2}, \ldots, a_{n}\right)$ by $f\left(U_{k}\right)=a_{k}$. It is easy to verify that $f$ is weakly singular and that $\alpha X \cong$ $w_{f}\left(X,\left\{a_{1}, a_{2}, \ldots, a_{n}\right\}\right)$.

We might note in passing that the assumption that every compactification with finite remainder is weakly singular implies Magill's Theorem.

In [6] W. W. Comfort considered spaces $X$ for which $\beta X \backslash X$ is a retract of $\beta X$. He showed that such spaces must be pseudocompact. In his proof Comfort used the Continuum Hypothesis. This dependence on $\mathrm{CH}$ was later removed by E. K. Van Douwen [17]. Another proof which was independent of $\mathrm{CH}$ was given by John B. Conway [7]. Conway's elegant proof used the, now classic, result of R. S. Phillips [14] that there is no bounded linear projection from $l_{\infty}\left(=C^{*}(\mathbf{N})\right)$ onto $c_{0}\left(=C_{\infty}(\mathbf{N})\right)$. Conway actually proved a result more general than that of Phillips. He proved that if there is a bounded linear projection from $C^{*}(X)$ onto $C_{\infty}(X)$, then $X$ must be pseudocompact. If $\beta X \backslash X$ is a retraction of $\beta X$, then the existence of such a projection is immediate. Conway also observed that a retraction may fail to exist even when a projection exists. The quotient space in Example 3 is an example of this. The following theorem partially resolves the question of how projections and retractions relate. A sizeable literature has grown up dealing with results in this form. See for example $[8,9$ 15].

THEOREM 2. Let $\alpha X$ be a compactification of $X$. Then $\alpha X \backslash X$ is a retract of $\alpha X$ if and only if there exists a projection $P: C_{\alpha}(X) \rightarrow C_{\infty}(X)$ whose complementary projection is a ring homomorphism.

PROOF. First let us suppose that such a projection exists. If $Q_{1}=I-P$, then by assumption $Q_{1}$ is a ring homomorphism. Let $\iota$ be the extension operator from $C_{\alpha}(X)$ to $C(\alpha X)$ and $\iota^{-1}$ its inverse, the operator which restricts a function in $C(\alpha X)$ to $X$. The mapping $Q=\iota \circ Q_{1} \circ \iota^{-1}$ is a bounded projection from $C(\alpha X)$ to $C(\alpha X)$ whose null space is

$$
\mathscr{N}(Q)=\left\{f \in C(\alpha X):\left.f\right|_{\alpha X \backslash X}=0\right\} .
$$

In addition $Q$ is also a ring homomorphism. For a subset $S$ of $\alpha X$ let us denote by $E_{S}$ the set of all evaluation functionals based on $S$. This is to say that $E_{S}=$ $\left\{x^{*} \in C(\alpha X)^{*}\right.$ : there exists an $s \in S$ so that $x^{*}(f)=f(s)$ for all functions $f \in C(\alpha X)\}$. Let us denote the evaluation functional at $s$ by $x_{s}^{*}$. It is well known $\left[10\right.$, p. 442] that $E_{S}$, endowed with the weak* topology, is homeomorphic to $S$ when $S$ is compact. Now $Q^{*}: C(\alpha X)^{*} \rightarrow C(\alpha X)^{*}$ is a projection, is weak continuous, and $E_{\alpha X \backslash X} \subseteq \mathscr{N}(Q)^{\perp}=\mathscr{R}\left(Q^{*}\right)$. From this it follows that $Q^{*}$ is the identity on $E_{\alpha X \backslash X}$. In fact $E_{\alpha X \backslash X}=\mathscr{R}\left(Q^{*}\right) \cap E_{\alpha X}$. Furthermore, if $x_{s}^{*} \in E_{\alpha X}$, then

$$
\begin{aligned}
Q^{*} x_{s}^{*}(f \cdot g) & =x_{s}^{*}(Q(f \cdot g))=x_{s}^{*}(Q f \cdot Q g)=Q f(s) \cdot Q g(s) \\
& =x_{s}^{*}(Q f) \cdot x_{s}^{*}(Q f)=Q^{*} x_{s}^{*}(f) \cdot Q^{*} x_{s}^{*}(g) .
\end{aligned}
$$

From this it follows $[\mathbf{1 2}$, p. 53] that the image of an evaluation functional under $Q^{*}$ is again an evaluation functional. From all that has been said it follows that $r=\left.Q^{*}\right|_{E_{\alpha X}}$ is a retraction from $E_{\alpha X}$ onto $E_{\alpha X \backslash X}$. Hence $\alpha X \backslash X$ is a retract of $\alpha X$, so that $\alpha X$ is singular. 
Conversely suppose that $\alpha X$ is singular. By Theorem 1 there is a retraction $r: \alpha X \rightarrow \alpha X \backslash X$. Define $Q: C(\alpha X) \rightarrow C(\alpha X)$ by $Q f=f$ or. Define $Q_{1}: C_{\alpha}(X) \rightarrow$ $C_{\alpha}(X)$ by $Q_{1}=\iota^{-1} \circ Q \circ \iota$. $Q_{1}$ is clearly a bounded linear projection which is a ring homomorphism. In addition $\mathscr{N}\left(Q_{1}\right)=C_{\infty}(X)$. The required projection is $P=I-Q_{1}$.

The following is a generalization of Phillip's theorem.

COROLLARY 3. Let $\alpha X$ be a singular compactification of $X$. If there exists a bounded linear projection from $C^{*}(X)$ onto $C_{\alpha}(X)$ then $X$ is pseudocompact.

PROOF. If such a projection exists then, by composition, there is a projection from $C^{*}(X)$ onto $C_{\infty}(X)$.

\section{REFERENCES}

1. George L. Cain, Jr., Compact and related mappings, Duke Math. J. 33 (1966), 639-645.

2. __ Mappings with prescribed singular sets, Nieuw. Arch. Wisk. (3) 17 (1969), 200-203.

3. George L. Cain, Jr., Richard E. Chandler, and Gary D. Faulkner, Singular sets and remainders, Trans. Amer. Math. Soc. 268 (1981), 161-171.

4. Richard E. Chandler, Gary D. Faulkner, Josephine P. Guglielmi, and Margaret Memory, Generalizing the Alexandroff-Urysohn double circumference construction, Proc. Amer. Math. Soc. 83 (1981), 606-608.

5. Richard E. Chandler and F. C. Tzung, Remainders in Hausdorff compactifications, Proc. Amer. Math. Soc. 70 (1978), 196-202.

6. W. W. Comfort, Retractions and other continuous maps from $\beta X$ to $\beta X \backslash X$, Trans. Amer. Math. Soc. 114 (1965), 1-9.

7. John B. Conway, Projections and retractions, Proc. Amer. Math. Soc. 17 (1966), 843-847.

8. D. W. Dean, Projections in certain continuous function spaces, Canad. J. Math. 14 (1962), 385-401.

9. $\ldots$ Subspaces of $C(H)$ which are direct factors of $C(H)$, Proc. Amer. Math. Soc. 16 (1965), 237-242.

10. N. Dunford and J. T. Schwartz, Linear operators, Wiley, New York, 1957.

11. Josephine P. Guglielmi, Compactifications with singular remainders, Ph.D. Thesis, North Carolina State Univ., 1986.

12. H. Elton Lacey, The isometric theory of classical Banach spaces, Springer-Verlag, New York, 1974.

13. K. D. Magill, Jr., N-point compactifications, Amer. Math. Monthly. Soc. 72 (1965), 10751081.

14. R. S. Phillips, On linear transformations, Trans. Amer. Math. Soc. 48 (1940), 516-541.

15. A. Sobczyk, Projection of the space $(m)$ on its subspace $\left(c_{0}\right)$, Bull. Amer. Math. Soc. $47(1941)$, 938-947.

16. G. T. Whyburn, Compactifications of mappings, Math. Ann. 166 (1966), 168-174.

17. Eric K. van Douwen, Retractions from $\beta X$ onto $\beta X \backslash X$, General Topology Appl. 9 (1978), 169-173.

Department of Mathematics, North Carolina State University, Raleigh, NORTH CAROLINA 27695-8205 